\title{
Which Pharmacovigilance for Vaccines?
}

\author{
Hervé Le Louët ${ }^{1}$, Elisabeth Loupi ${ }^{2}$, Françoise Haramburu ${ }^{3}$ and the participants in Round Table $N^{\circ} 3$, \\ Giens XXII^
}

1 Service de Pharmacologie, Hôpital Henri Mondor, Paris, France

2 sanofi pasteur, Lyon, France

3 Service de Pharmacologie, Hôpital Pellegrin Carreire, Bordeaux, France

\begin{abstract}
Keywords:
vaccines;

pharmacovigilance;

epidemiology

Abstract - Vaccines are not separate health products but anti-infectious medicines administered for the large part prophylactically and for which the effect is immunological and not pharmacological. They should be evaluated by the usual methods of clinical pharmacology and pharmacovigilance, taking into account certain specificities (mechanism of action, manufacture, frequent administration to healthy subjects, particular recommendations, etc.).

Experience from some vaccination campaigns have revealed insufficiencies notably in data collection allowing evaluation of the interest of a vaccine to public health, its relevance to the recent epidemiology of the disease in question and long-term security. The absence of data can generate fear in the general population that is broadcast by anti-vaccination lobby.

For a more optimal pharmacovigilance of vaccines, it is necessary to: (i) improve the coherence between the evaluating authorities; (ii) set up, in addition to the usual risk management plan, an active microbiological and epidemiological surveillance and to follow up exposed populations; (iii) have programmes of education of the medical community regarding vaccination and health education for the general public.
\end{abstract}

\section{Introduction}

Considered for some time as different to medicines, vaccines fit the French definition of the latter by their function: "any substance or composition of substances with curative or preventative properties for human or animal diseases, as well as any substance or combination of substances that can be used on or administered to humans or animals to establish a medical diagnostic; to restore correct or modify their physiological functions or that exert a pharmacological, immunological or metabolic action" ${ }^{[1]}$ Despite this, the vaccine is perceived in a particular way by the society which does no fully appreciate the diseases concerned, their seriousness (mortality/morbidity) or the epidemiological context. Several factors can lead to a poor appreciation of the benefits and risks of vaccines.

As described above, vaccines are not separate health products but anti-infectious medicines administered for the large part prophylactically and for which the effect is immunological and not pharmacological as defined in the European directive 2001/83/CE. ${ }^{[2]}$ For this reason, they should be evaluated by the

\footnotetext{
* For a list of participants, please see the end of the article.
}

usual methods of clinical pharmacology and pharmacovigilance. However, taking into account certain specificities described further down, vaccines are the subject of particular recommendations and regulation in several countries notably in North America ${ }^{[3,4]}$ but also in Europe where documents regarding clinical trials ${ }^{[5]}$ and pharmacovigilance ${ }^{[6]}$ have been published.

\section{Vaccines and their particularities}

\section{1. Mechanism of action}

The mechanism of action is still not completely resolved. It does differ however from a pharmacological effect as the substance is not required to be present to elicit the effect and by the persistence of this effect which is mediated by a complex cascades formed of multiple and heterogeneous targets.

\subsection{Manufacture}

The vaccine is a biological drug, the result of high technology but also both operator- and ingredient-dependent. The manufacture of vaccines uses strict protocols and quality-control. 
Any modification in the manufacturing procedure, supplier of excipients or adjuvants or active ingredients can induce in the final product modifications in its stability and even its efficacy that can explain some supply problems.

\subsection{Use}

Most commonly vaccines are administered to healthy subjects and often to neonates and children. Vaccines are the subjects of double prescription, that is to say by both the physician and by the authorities and follows an immunisation schedule that varies between different countries (there exists at the time of writing more than 20 different schemes in the European countries). ${ }^{[7]}$ This leads to a situation where in some countries a vaccination is compulsory whereas in others this is not the case but strongly recommended. For example in France vaccinations against diphtheria, tetanus and poliomyelitis are compulsory ${ }^{[8]}$ whereas in Great Britain these are only highly recommended and the population is incited to undergo vaccination by communication through the media (television).

Certain vaccines are administered in specialised centres, for example rabbies and yellow fever vaccines. Vaccination campaigns can be organised for example in developing countries (vaccination against yellow fever in Ivory Coast for example) or as an emergency measure in the case of epidemics (meningitis $\mathrm{C}$ in some areas of France).$^{[9,10]}$

\subsection{Impact}

The vaccine protects both the individual and the society, modifying the dynamic of an epidemic, reducing the circulation of pathogens ${ }^{[11,12]}$ or favouring the appearance of new serotypes. ${ }^{[13]}$

Vaccination can be adapted to the epidemiology of the micro organisms in function of their evolution, for example annual influenza variation and geographical meningitis variation.

\section{Pharmacovigilance}

Adverse events occurring after vaccination are less well accepted than those associated with other medicines. Today, we vaccinate against diseases that are less well-known by both the physicians and the patients and the benefit of such an act is not always immediately perceivable. In the situation of a vaccination campaign and taken into account the large number of vaccines over a short period of time, it is important to collect all adverse events. A cluster of these can indicate a production or administration error and should be rapidly fully investigated. Data collection can be aided by using special forms that can be used in normal practice as well as during vaccination campaigns.

As with other medicines, spontaneous reporting is the most frequently used method of detection for adverse events. However, for vaccines, the analysis case-by-case or with a series of cases using classical causality assessment methods is most often not very useful. Only local or immediate adverse effects or reactions that occur after administration of attenuated virus vaccines can be attributed with a degree of confidence to the vaccination. Thus, clinical and epidemiological studies are required to confirm this signal. Indeed, criteria usually used to determine causality such as evolution of the event following treatment discontinuation and the result of re-challenge cannot, for the most part, be used to assess causality of an event occurring after vaccination. This is due to the mechanism of action of a vaccine that once administered, triggers a cascade of immunological events which are not controllable. It is necessary to assess the risk, taking into account the epidemiological and clinical characteristics of the diseases that can occur in the age range of the population targeted by the vaccine.

\section{Communication}

Communication, which is essential to the domain of vaccines, especially in periods of crisis, does not differ from other health communication in general in its objectives and modalities. It should however be of excellent quality and call upon professionals from the required disciplines (communication, science, epidemiology, sociology, physicians etc.). The loss of confidence in a vaccination campaign plays an important role in public health as can be judged from the proceeding examples.

\section{Lessons from the past}

Experience from several vaccination campaigns have revealed insufficiencies notably in data collection allowing evaluation of the interest of a vaccine to public health, its relevance to the recent epidemiology of the disease in question and long-term security. To avoid this, it is necessary to equip oneself with measures to know: when and why and when to vaccinate, targeting the population at risk and with what risks. The absence of data can generate fear in the general population that is broadcast by antivaccination lobby. This has been the case in France for multiple sclerosis and hepatitis B vaccine or macrophagic myofasciitis and vaccines containing an aluminium adjuvant and in Great Britain autism and vaccine for the measles-mumps-rubella.

Although there exists methods to evaluate efficacy to enable market authorisation, this is not the case for the measurement of the impact the vaccine has on the population and there generally 
does not exist data to evaluate the long-term safety and effectiveness of the vaccine. Furthermore, there often lacks harmony between recommendations issued by the authorities in France, i.e. Afssaps (benefits risks ratio) the Comité Technique des Vaccinations (infectious disease prevention strategy, utility and annual recommendations) and the Haute Autorité de Santé (therapeutic strategy, effectiveness, efficiency, utility).

\section{Propositions}

1. Improvement of the coherence between the evaluating authorities: joint meetings and opinions of the "Comité Technique des Vaccinations" and the "Haute Autorité de Santé" ("commission de la transparence"); consultation of the firms before launching. Recently inter-institution consultation has taken place yet, vaccines should not be commercialised before the health authorities pass judgement.

2. In addition to the measures already taken in a risk management strategy:

- there needs to be an active microbiological and epidemiological surveillance by reinforcing and enlarging the role of existing structures (For example in France the "Centre Nationaux de Référence" [CNR], "Institut de Veille Sanitaire") and creating those necessary (CNR for bacteria and viruses concerned by vaccination campaigns, setting up of registers);

- data must be made available to evaluate pharmacovigilance of exposed populations to improve analysis and reactivity in the face of an emerging risk: epidemiological data for diseases likely to occur in the vaccinated population (cross-referencing of files present in different databases)

3. Education of the medical community regarding vaccination and health education for the general public should be reinforced.

These concrete measures that are applicable in the short-term are indispensable for the optimisation of vaccine use and to the improvement of security as perceived by the general population.

\section{Participants}

Atika Abelin (Sanofi Pasteur), Didier Guillemot (Centre Ressources Biostatistiques, Pasteur, Paris), Françoise Haramburu
(Hôpital Pellegrin Carreire, Bordeaux), Alexis Jacquet (Afssaps), Carmen Kreft-Jais (Afssaps), Odile Launay (Groupe Hospitalier Cochin Saint Vincent de Paul, Paris), Hervé Le Louët (Hôpital Henri Mondor, Paris), Elisabeth Loupi (Sanofi Pasteur), Muriel Malbezin (GlaxoSmithKline)

\section{References}

1. Code de la santé publique. Art L-5111-1

2. Directive 65/65/CEE du Conseil, du 26 janvier 1965, concernant le rapprochement des dispositions législatives, réglementaires et administratives, relatives aux spécialités pharmaceutiques

3. USA / FDA. CFR (Code of Federal Regulation) Title 21: Food and drugs. Chapter 1: Food and Drug administration. Subchapter F: Biologics. Part 600: Biological products : General

4. Canada / PPHB. Guidelines for reporting adverse events associated with vaccine products. Canada Communicable Disease Report. Volume 26 - S1 February 2000

5. Guideline on clinical evaluation of new vaccines EMEA/CHMP/VWP/382702/2006, October 2006

6. Concept paper for a guideline on the conduct of pharmacovigilance for vaccines. PhVWP/372004/2005 - October 2005

7. www.ecdc.eu.int/Health_topics/VI/VI_newsletter.html

8. Anon. Calendrier vaccinal 2006 ; avis du Conseil Supérieur d'Hygiène publique de France. BEH N N $^{\circ} 930 / 2006$

9. Laribière A, Miremont-Salamé G, Reyre H, et al. Surveillance of adverse effects during a vaccination campaign against meningitis $\mathrm{C}$. Eur J Clin Pharmacol. 2005; 61: 907-11

10. Bagheri H, Gony M, Montastruc JL. A propos d'une campagne de vaccination contre la méningite $\mathrm{C}$ dans les Hautes-Pyrénées : réflexions de pharmacovigilance. Therapie 2005; 60: 287-94

11. Anon. Direct and Indirect effects of routine vaccination of children with 7 valent pneumococcal conjugate vaccine on incidence of invasive pneumococcal disease - United States, 1998-2003. PPR weekly 2005; 54: 893-7

12. Metlay JP, Fishman NO, Joffe M, et al. Impact of pediatric vaccination with pneumococcal conjugate vaccine on the risk of bacteremic pneumococcal pneumonia in adults. Vaccine 2006; 24: 468-75

13. Law DKS, Lorange M, Ringuette L, et al. Invasive meningococcal disease in Quebec, Canada, due to an emerging clone of ST-269 serogroup B meningococci with serotype antigen 17 and serosubtype antigen P1.19 (B:17:P1.P19). J Clin Microbiol 2006; 44: 2743-9

Correspondence and offprints: Françoise Haramburu, Service de Pharmacologie, Hôpital Pellegrin Carreire, 146 rue Léo Saignat, 33076 Bordeaux Cedex, France.

E-mail: francoise.haramburu@pharmaco.u-bordeaux2.fr 